\title{
61. Sur la Dérivation de l'Intégrale (E. R.) Indéfinie. II
}

\section{Par Shizu NAKANishi}

Institut des Mathématiques, Université d'Osaka

(Comm. by K. KunUGi, M.J.A., May 13, 1958)

Continuons l'étude sur la dérivation de l'intégrale (E. R.) indéfinie. ${ }^{1)}$

Théorème 8. Soit $u=\left\{u_{n}\right\}, u_{n}=V\left(F_{n}, \nu_{n} ; f_{n}\right)$, une suite fondamentale satisfaisant aux conditions $\left[3_{1}\right]$ et $\left[3_{2}\right]$. Posons $f(x)=\lim _{n \rightarrow \infty} f_{n}(x)$. Alors, l'intégrale (E. R.) indéfinie $F(x)$ de $f(x)$ est une fonction absolument continue au sens large ${ }^{2)}$ sur tout $F_{n}^{*}$, ò̀ $F_{n}^{*}=\bigcap_{m=n}^{\infty} F_{m},(n=0$, $1,2, \cdots)$.

En effet, puisqu'on a

$$
F(x)=\int_{\alpha}^{x} f_{0}(x) d x+\int_{\alpha}^{x} h(x) d x+\sum_{n=0}^{\infty} \int_{a}^{x} r_{n}(x) d x,
$$

où $h(x)=\sum_{n=0}^{\infty} p_{n}(x)$, il suffit de montrer que la fonction $G(x)=\sum_{n=0}^{\infty} \int_{\alpha}^{x} r_{n}(x) d x$ est absolument continue au sens large sur tout $F_{m}^{*}$. Soient $\varepsilon$ un nombre positif quelconque et $n_{0}$ une entier positif tel que $\sum_{i=n_{0}+1}^{\infty} 2^{-\nu_{i}}<\varepsilon / 2$ et $n_{0}>m$. Alors, l'intégrale de la fonction $\sum_{i=0}^{n_{0}}\left|r_{i}(x)\right|$ étant absolument continue sur $[a, b]$, il existe un nombre positif $\delta=\delta(\varepsilon)$ tel que

$$
\text { mes } E<\delta \text { entraîne } \int_{E}\left(\sum_{i=0}^{n_{0}}\left|r_{i}(x)\right|\right) d x<\frac{\varepsilon}{2} \text {, }
$$

quel que soit l'ensemble $E$ contenu dans $[a, b]$.

Soit $\left[a_{j}, b_{j}\right]\left(j=1,2, \cdots, j_{0}\right)$ un système élémentaire quelconque tel que les extrémités appartiennent à $F_{m}^{*}$ et dont la mesure $\sum_{j=1}^{j_{0}}\left(b_{j}-a_{j}\right)$ est inférieur à $\delta$. Puisqu'alors les extrémités des intervalles $\left[a_{j}, b_{j}\right]$ appartiennent à $F_{i}$ pour tout $i \geq n_{0}+1$, on a, d'après la condition $\left[3_{2}\right]$, pour tout $i \geq n_{0}+1$

Donc, on a

$$
\sum_{j=1}^{j_{0}}\left|\int_{\alpha_{j}}^{b_{j}} r_{i}(x) d x\right|<2^{-\nu_{i}} .
$$

$$
\begin{aligned}
\sum_{j=1}^{j_{0}}\left|G\left(b_{j}\right)-G\left(a_{j}\right)\right| & \leq \sum_{j=1}^{j_{0}} \int_{\alpha_{j}}^{b_{j}}\left(\sum_{i=0}^{n_{0}}\left|r_{i}(x)\right|\right) d x+\sum_{j=1}^{j_{0}} \sum_{i=n_{0}+1}^{\infty}\left|\int_{a_{j}}^{b_{j}} r_{i}(x) d x\right| \\
& <\frac{\varepsilon}{2}+\sum_{i=n_{0}+1}^{\infty} 2^{-\nu_{i}}<\frac{\varepsilon}{2}+\frac{\varepsilon}{2}=\varepsilon .
\end{aligned}
$$

1) S. Nakanishi: Sur la dérivation de l'intégrale $(E . R$.) indéfinie. I, Proc. Japan Acad., 34, 199-204 (1958). (1937).

2) Pour la définition, voir, par exemple, S. Saks: Theory of the Integral, 223 
De plus, on en déduit le

Corollaire 1. Soit $u=\left\{u_{n}\right\}, u_{n}=V\left(F_{n}, \nu_{n} ; f_{n}\right)$, une suite fondamentale satisfaisant aux conditions $\left[3_{1}\right]$ et $\left[3_{2}\right]$. Posons $f(x)=\lim _{n \rightarrow \infty} f_{n}(x)$. Alors, l'intégrale (E. R.) indéfinie de $f(x)$ est absolument continue généralisée au sens large ${ }^{3)}$ sur l'ensemble $\bigcup_{n=0}^{\infty} F_{n}^{*}$, où $F_{n}^{*}=\bigcap_{m=n}^{\infty} F_{m}$.

Montrons ensuite quelques conditions qui sont suffisantes pour que l'intégrale $(E . R$.) indéfinie $F(x)$ remplisse la condition $(N)$ de Lusin sur $[a, b]$, c.-à-d. pour tout ensemble $E$ contenu dans $[a, b]$ mes $E=0$ entraîne mes $[F(x) ; x \in E]=0$.

Or, $F(x)$ remplit, en vertu du Corollaire 1, la condition $(N)$ de Lusin sur $\bigcup_{n=0}^{\infty} F_{n}^{*}$. Donc, il suffit de montrer que les conditions entraînent la propriété suivante:

$$
\operatorname{mes}\left[F(x) ; x \in[a, b]-\bigcup_{n=0}^{\infty} F_{n}^{*}\right]=0 .
$$

Lemme 1. Soit $u=\left\{u_{n}\right\}, u_{n}=V\left(F_{n}, \nu_{n} ; f_{n}\right)$, une suite fondamentale satisfaisant aux conditions $\left[3_{1}\right]$ et $\left[3_{2}\right]$ et tel qu'il existe une fonction $\phi(n)$ de $n(n=0,1,2, \cdots)$ qui jouit des conditions suivantes:

$$
\begin{gathered}
\phi(n)>0 \quad \text { pour } n=0,1,2, \cdots ; \\
\sum_{n=0}^{\infty} \phi(n)<\infty ;
\end{gathered}
$$

(3) pour tout ensemble $E$ contenu dans $[a, b]-F_{n}$, on $a$

$$
\int_{E}\left|f_{n+1}(x)\right| d x \leq \phi(n) \text {. }
$$

Posons $f(x)=\lim _{n \rightarrow \infty} f_{n}(x)$. Alors, $F(x)=\left(E . R\right.$.) $\int_{a}^{x} f(x) d x$ possède la propriété suivante:

$$
\operatorname{mes}\left[F(x) ; x \in[a, b]-\bigcup_{n=0}^{\infty} F_{n}^{*}\right]=0, \text { ò̀ } F_{n}^{*}=\bigcap_{m=n}^{\infty} F_{m} .
$$

Démonstration. Posons $A^{*}=[a, b]-\bigcup_{n=0}^{\infty} F_{n}^{*}$ et $G_{n}^{*}=[a, b]-F_{n}^{*}$. On a alors $A^{*}=\bigcap_{n=0}^{\infty} G_{n}^{*}$. Soit $\varepsilon$ un nombre positif quelconque. Alors, il existe un entier positif $n_{0}=n_{0}(\varepsilon)$ jouissant des propriétés suivantes:

$$
\begin{aligned}
& 2^{-\nu_{n_{0}}+4}<\varepsilon / 10 ; \\
& \sum_{n=n_{0}}^{\infty} \phi(n)<\varepsilon / 10 ;
\end{aligned}
$$

3)

$$
\int_{G_{n_{0}}^{*}} p^{*}(x) d x<\varepsilon / 10 \text {, où } p^{*}=\sum_{n=0}^{\infty}\left|p_{n}(x)\right| ;
$$

4) si $\left|x_{1}-x_{2}\right|<\operatorname{mes}\left(G_{n_{0}}^{*}\right)$, on a $\left|F\left(x_{1}\right)-F\left(x_{2}\right)\right|<\varepsilon / 10$. $G_{n_{0}}^{*}$ étant un ensemble ouvert dans $[a, b]$, il s'exprime par la somme de la suite finie ou dénombrable d'intervalles disjoints. Désignons par

3) Pour la définition, voir S. Saks: Loc. cit., 223. 
$I_{n_{0} i}=\left(a_{n_{0} i}, b_{n_{0} i}\right)(i=1,2, \cdots)$ tous les intervalles de la suite qui contiennent au moins un point de $A^{*}$ et tels que $a_{n_{0} i} \neq a$ ou $b_{n_{0} i} \neq b$, et par $\left[a, b_{0}\right)$ et $\left(a_{0}, b\right]$ les intervalles qui contiennent respectivement $a$ et $b$ (si'il existe).

A) Montrons d'abord qu'on a, quel que soit $x_{i} \in I_{n_{0} i} \cap A^{*}(i=1$, $2, \cdots), \sum_{i=1}^{\infty}\left|F\left(x_{i}\right)-F\left(a_{n_{0} i}\right)\right|<3 \varepsilon / 10$.

Puisque $G_{n}^{*}$ s'exprime la somme de la suite finie ou dénombrable disjoints et que $x_{i}(i=1,2, \cdots)$ appartiennent à $G_{n}^{*}$, on a, pour tout $n \geq n_{0}+1$, les intervalles de la suite $I_{n i}=\left(a_{n i}, b_{n i}\right)(i=1,2, \cdots)$ tels que $I_{n i} \cap I_{n i^{\prime}}=0\left(i \neq i^{\prime}\right)$ et $I_{n i} \ni x_{i}$. Soit, pour tout $n \geq n_{0}+1, I_{n i j}(j=1,2, \cdots)$ tous les intervalles de la suite contenus dans $\left(a_{n-1, i}, a_{n i}\right)(i=1,2, \cdots)$. Alors, les intervalles possèdent les propriétés suivantes:

$$
I_{n_{0} i} \supseteq I_{n_{0}+1, i} \supseteq \cdots \text { et } \bigcap_{n=n_{0}}^{\infty} I_{n i}=\left\{x_{i}\right\} \text {. }
$$

6) les extrémités $a_{n i}$ et $b_{n i}$ des intervalles $I_{n i}\left(n \geq n_{0}\right)$ appartiennent à $F_{n}^{\prime *}$, et on a $I_{n i} \cap F_{n}^{*}=0$;

7) les extrémités des intervalles $I_{n i j}\left(n \geq n_{0}+1\right)$ appartiennent à $F_{n}^{*}$, et on a $I_{n i j} \cap F_{n}^{*}=0$ et $\bigcup_{j=1}^{\infty} I_{n i j}=\left(a_{n-1, i}, a_{n i}\right) \cap G_{n}^{*}$.

Ceci établi, nous allons montrer qu'on a $\sum_{i=1}^{\infty}\left|F\left(x_{i}\right)-F\left(a_{n_{0}}\right)\right|<3 \varepsilon / 10$. Puisqu'on a $\bigcup_{i=1}^{\infty}\left(a_{n i}, a_{n+1, i}\right) \subseteq G_{n}^{*} \subseteq[a, b]-F_{n}$, on a d'abord, d'après (3), pour tout $n \geq n_{0}$

$$
\sum_{i=1}^{\infty} \int_{\alpha_{n i}}^{a_{n+1, i}}\left|f_{n+1}(x)\right| d x \leq \phi(n) .
$$

De plus, puisque les extrémités des intervalles $I_{n+1, i j}$ appartiennent à $F_{n+1}^{*}$ et qu'elles appartiennent donc à $F_{m}$ pour tout $m \geq n+1$, on a, d'après $\left[3_{2}\right]$, pour tout $m \geq n+1$,

$$
\sum_{i=1}^{\infty} \sum_{j=1}^{\infty}\left|\int_{I_{n+1, i j}} r_{m}(x) d x\right| \leq 2^{-\nu_{m}},
$$

d'où, en notant que $r_{m}(x)$ s'annule pour tout $x$ appartenant à $F_{n+1}^{*}$ pour tout $m \geq n+1$, on conclut que

$$
\begin{aligned}
\sum_{i=1}^{\infty} \sum_{m=n+1}^{\infty}\left|\int_{\boldsymbol{a}_{n i}}^{a_{n+1, i}} r_{m}(x) d x\right| & =\sum_{m=n+1}^{\infty}\left(\sum_{i=1}^{\infty}\left|\int_{\alpha_{n i}}^{a_{n+1, i}} r_{m}(x) d x\right|\right) \\
& \leq \sum_{m=n+1}^{\infty}\left(\sum_{i=1}^{\infty} \sum_{j=1}^{\infty}\left|\int_{I_{n+1, i j}} r_{m}(x) d x\right|\right) \\
& \leq \sum_{m=n+1}^{\infty} 2^{-\nu_{m}<2^{-\nu_{n+1}+2}} .
\end{aligned}
$$

Mais comme l'intégrale $(E . R$.) indéfinie $F(x)$ s'écrit

$$
F(x)=\int^{x} f_{n+1}(x) d x+\int_{a}^{x} h_{n+1}(x) d x+\sum_{m=n+1}^{\infty} \int_{a}^{x} r_{m}(x) d x,
$$


où $h_{n+1}(x)=\sum_{m=n+1}^{\infty} p_{m}(x)$, on a pour tout $n \geq n_{0}$

$$
\begin{aligned}
& \sum_{i=1}^{\infty}\left|F\left(a_{n+1, i}\right)-F\left(a_{n i}\right)\right| \\
\leq & \sum_{i=1}^{\infty} \int_{a_{n i}}^{a_{n+1, i}}\left|f_{n+1}(x)\right| d x+\sum_{i=1}^{\infty} \int_{a_{n i}}^{a_{n+1, i}}\left|h_{n+1}(x)\right| d x+\sum_{i=1}^{\infty} \sum_{m=n+1}^{\infty}\left|\int_{a_{n i}}^{a_{n+1, i}} r_{m}(x) d x\right| \\
< & \phi(n)+\sum_{i=1}^{\infty} \int_{a_{n i}}^{a_{n+1, i}} p^{*}(x) d x+2^{-\nu_{n+1}+2} .
\end{aligned}
$$

Or, $F(x)$ étant continue, on a pour tout $i$

Donc, on a

$$
F\left(x_{i}\right)-F\left(a_{n_{0} i}\right)=\sum_{n=n_{0}}^{\infty}\left(F\left(a_{n+1, i}\right)-F\left(a_{n i}\right)\right) .
$$

$$
\begin{aligned}
\sum_{i=1}^{\infty}\left|F\left(x_{i}\right)-F\left(a_{n_{0} i}\right)\right| & \leq \sum_{i=1}^{\infty} \sum_{n=n_{0}}^{\infty}\left|F\left(a_{n+1, i}\right)-F\left(a_{n i}\right)\right| \\
& \leq \sum_{n=n_{0}}^{\infty} \phi(n)+\sum_{n=n_{0}}^{\infty} \sum_{i=1}^{\infty} \int_{a_{n i}}^{a_{n+1, i}} p^{*}(x) d x+\sum_{n=n_{0}}^{\infty} 2^{-\nu_{n+1}+2} \\
& <\frac{\varepsilon}{10}+\int_{a_{n_{0}}^{*}} p^{*}(x) d x+2^{-\nu_{n_{0}+1}+4}<\frac{3}{10} \varepsilon .
\end{aligned}
$$

B) On a maintenant mes $\left[F(x) ; x \in A^{*}\right] \leq \varepsilon$. En effet, si l'on pose

$$
\begin{array}{cl}
l_{i}=\max _{x}\left(\left|F\left(a_{n_{0} i}\right)-F(x)\right| ; x \in A^{*}\right. & \text { et } \left.x \in\left(a_{n_{0} i}, b_{n_{0} i}\right)\right), \\
J_{i}=\left[F\left(a_{n_{0} i}\right)-l_{i}, F\left(a_{n_{0} i}\right)+l_{i}\right] & (i=1,2, \cdots),
\end{array}
$$

on a d'abord d'après 4)

$$
\begin{aligned}
\left\{\bigcup _ { i = 1 } ^ { \infty } J _ { i } \bigcup \left[F(a)-\frac{\varepsilon}{10},\right.\right. & \left.\left.F(a)+\frac{\varepsilon}{10}\right] \bigcup\left[F(b)-\frac{\varepsilon}{10}, F(b)+\frac{\varepsilon}{10}\right]\right\} \\
& \supseteq\left[F(x) ; x \in A^{*}\right],
\end{aligned}
$$

puisque $A^{*} \subseteq \bigcup_{i=1}^{\infty}\left(a_{n_{0} i}, b_{n_{0}}\right) \cup\left[a, b_{0}\right) \cup\left(a_{0}, b\right]$. D'autre part, puisqu'il existe, pour $\eta>0$ arbitraire, un point $x_{i}$ de $A^{*}$ appartenant à $\left(a_{n_{0} i}, b_{n_{0} i}\right)$ et tel que $l_{i}-\frac{\eta}{2^{i}}<\left|F\left(a_{n_{0} i}\right)-F\left(x_{i}\right)\right|$, on a d'après $A$ )

$$
\sum_{i=1}^{\infty} l_{i}<\sum_{i=1}^{\infty}\left|F\left(a_{n_{0} i}\right)-F\left(x_{i}\right)\right|+\sum_{i=1}^{\infty} \frac{\eta}{2^{i}}<\frac{3}{10} \varepsilon+\eta
$$

et on a par suite $\sum_{i=1}^{\infty} l_{i} \leq \frac{3}{10} \varepsilon$. Donc, il en résulte qu'on a mes $[F(x)$; $\left.x \in A^{*}\right] \leq 2\left(\sum_{i=1}^{\infty} l_{i}\right)+\frac{4}{10} \varepsilon \leq \varepsilon$.

Corollaire 2. Soit $u=\left\{u_{n}\right\}, u_{n}=V\left(F_{n}, \nu_{n} ; f_{n}\right)$, une suite fondamentale satisfaisant aux conditions $\left[3_{1}\right]$ et $\left[3_{2}\right]$ et qui possède la propriété $P^{*} .^{4)}$ En outre, supposons que pour la propriété $P^{*}$ la fonction $\phi(n)$ de $n$ jouisse de la propriété plus forte $\sum_{n=0}^{\infty} \phi(n)<\infty$. Alors, pour

4) Voir K. Kunugi: Application de la méthode des espaces rangés à la théorie de l'intégration. I, Proc. Japan Acad., 32, 215-220 (1956). 
l'intégrale (E. R.) indéfinie $F(x)$ de $f(x)=\lim _{n \rightarrow \infty} f_{n}(x)$, on a $\operatorname{mes}\left[F(x) ; x \in[a, b]-\bigcup_{n=0}^{\infty} F_{n}^{*}\right]=0$, où $F_{n}^{*}=\bigcap_{m=n}^{\infty} F_{m}$.

Ensuite, considérons la condition suivante plus forte $\left[3_{2}^{*}\right]$ au lieu de la condition $\left[3_{2}\right]$.

$\left[3_{2}^{*}\right]$ Pour tout système élémentaire d'intervalles $I_{i}(i=1,2, \cdots$, $i_{0}$ ) tels que l'un au moins des extrémités de $I_{i}$ appartient à $F$ pour tout $i$, on a

$$
\sum_{i=1}^{i_{0}}\left|\int_{I_{i}} r(x) d x\right|<\mathbf{2}^{-\nu}
$$

Nous pouvons démontrer alors le

Lemme 2. Soit $u=\left\{u_{n}\right\}, u_{n}=V\left(F_{n}, \nu_{n} ; f_{n}\right)$, une suite fondamentale satisfaisant aux conditions $\left[3_{1}\right]$ et $\left[3_{2}^{*}\right]$ et tel qu'il existe une fonction $\phi(n)$ de $n(n=0,1,2 \cdots)$ qui jouit des conditions suivantes:

$$
\begin{gathered}
\phi(n)>0 \quad \text { pour } n=0,1,2, \cdots ; \\
\sum_{n=0}^{\infty} \phi(n)<\infty ;
\end{gathered}
$$

pour tout ensemble $E$ contenu dans $[a, b]-F_{n}$, on $a$

$$
\int_{E}\left|f_{n}(x)\right| d x \leq \phi(n) .
$$

Posons $f(x)=\lim _{n \rightarrow \infty} f_{n}(x)$. Alors, pour l'intégrale (E. R.) indéfinie de $f(x), F(x)=(E . R$. $) \int_{a}^{x} f(x) d x$, on a

$$
\operatorname{mes}\left[F(x) ; x \in[a, b]-\bigcup_{n=0}^{\infty} F_{n}^{*}\right]=0 \text {, où } F_{n}^{*}=\bigcap_{m=n}^{\infty} F_{m} .
$$

Démonstration. Étant donné un $\varepsilon>0$ arbitraire, définisons, de même que Lemme 1 , un entier positif $n_{0}$ et, pour tout $n \geq n_{0}$, des suites d'intervalles

$$
\begin{gathered}
I_{n i}=\left(a_{n i}, b_{n i}\right)(i=1,2, \cdots), \\
I_{n+1, i j}(i=1,2, \cdots ; j=1,2, \cdots) .
\end{gathered}
$$

Alors, on a, quel que soit $x_{i} \in I_{n_{0} i} \cap A^{*}(i=1,2, \cdots), \sum_{i=1}^{\infty}\left|F\left(x_{i}\right)-F\left(a_{n_{0} i}\right)\right|$ $<3 \varepsilon / 10$. En effet, puisqu'on a $\bigcup_{i=1}^{\infty}\left(a_{n i}, a_{n+1, i}\right) \subseteq[a, b]-F_{n}$, on a d'abord, d'après (3), pour tout $n \geq n_{0}$

$$
\sum_{i=1}^{\infty} \int_{a_{n i}}^{a_{n+1, i}}\left|f_{n}(x)\right| d x \leq \phi(n)
$$

D'ailleurs, puisque l'extrémité $a_{n i}$ d'intervalle $\left[a_{n i}, a_{n+1, i}\right](i=1,2, \cdots)$ appartient à $F_{n}^{*}$ et qu'elle appartient donc à $F_{n}$, on a d'après [3 $\left.3_{2}^{*}\right]$ pour tout $n \geq n_{0}$

$$
\sum_{i=1}^{\infty}\left|\int_{a_{n i}}^{a_{n+1, i}} r_{n}(x) d x\right| \leq 2^{-\nu_{n}}
$$

On a de plus, de même que Lemme 1 , 


$$
\sum_{i=1}^{\infty} \sum_{m=n+1}^{\infty}\left|\int_{a_{n i}}^{a_{n+1, i}} r_{n}(x) d x\right| \leq \sum_{m=n+1}^{\infty} 2^{-\nu_{m}}
$$

Mais comme $F(x)$ s'écrit

$$
F(x)=\int_{a}^{x} f_{n}(x) d x+\int_{a}^{x} h_{n}(x) d x+\sum_{m=n}^{\infty} \int_{a}^{x} r_{m}(x) d x,
$$

où $h_{m}(x)=\sum_{m=n}^{\infty} p_{m}(x)$, on a pour tout $n \geq n_{0}$

$$
\begin{aligned}
& \sum_{i=1}^{\infty}\left|F\left(a_{n+1, i}\right)-F\left(a_{n i}\right)\right| \\
\leq & \sum_{j=1}^{\infty} \int_{a_{n i}}^{a_{n+1, i}}\left|f_{n}(x)\right| d x+\sum_{i=1}^{\infty} \int_{a_{n i}}^{a_{n+1, i}}\left|h_{n}(x)\right| d x+ \\
\leq & \phi(n)+\sum_{i=1}^{\infty} \int_{a_{n i}}^{a_{n+1, i}}\left|\int_{a_{n i}}^{a_{n+1}} r_{n}(x) d x\right|+\sum_{i=1}^{\infty} \sum_{m=n+1}^{\infty} \mid \int_{\alpha_{n i}}^{a_{n+1, i}} r_{m}(x) d x+2^{-\nu_{n}}+\sum_{m=n+1}^{\infty} 2^{-\nu_{m}} .
\end{aligned}
$$

Par conséquent, on a

$$
\sum_{i=1}^{\infty}\left|F\left(x_{i}\right)-F\left(a_{n_{0} i}\right)\right| \leq \sum_{i=1}^{\infty} \sum_{n=n_{0}}^{\infty}\left|F\left(a_{n+1, i}\right)-F\left(a_{n i}\right)\right|<3 \varepsilon / 10,
$$

d'où, de même que Lemme 1 , on peut conclure qu'on a mes $[F(x)$; $\left.x \in A^{*}\right]<\varepsilon$.

Nous pouvons déduire, de plus, le Théorème suivant de même que S. Saks: Theory of the Integral (1937), (6.2) Theorem, Chap. VII, $\S 6$, par exemple.

Théorème 9. Soient $u=\left\{u_{n}\right\}, u_{n}=V\left(F_{n}^{1}, \nu_{n}^{1} ; f_{n}\right)$ et $v=\left\{v_{n}\right\}, v_{n}=$ $V\left(F_{m}^{2}, \nu_{m}^{2} ; g_{m}\right)$ deux suites fondamentales qui jouissent d'un des hypothèses des Lemme 1, Théorème 1 et Lemme 2, et telles que l'on ait presque partout

alors, on a

$$
\lim _{n \rightarrow \infty} f_{n}(x)=\lim _{m \rightarrow \infty} g_{m}(x),
$$

$$
\lim _{n \rightarrow \infty} \int_{a}^{b} f_{n}(x) d x=\lim _{m \rightarrow \infty} \int_{a}^{b} g_{m}(x) d x .
$$

